\title{
Qualitätsziele der Spezialärztlichen Versorgung
}

\section{HANS-FRIEDRICH SPIES}

Dr. med. Hans-Friedrich Spies ist Internist - Kardiologe und Vizepräsident des Berufsverbandes Deutscher Internisten e.V. (BDI e. V.) in Wiesbaden

\author{
Die Qualitätsziele der ambulant spezialfachärztlichen \\ Versorgung (ASV) müssen vom Gemeinsamen \\ Bundesausschuss (G-BA) definiert werden. Die Aufgabe ist \\ sehr komplex und stellt dabei höchste Anforderungen an \\ dessen medizinischen Sachverstand. Der folgende Beitrag \\ setzt sich kritisch mit den Anforderungen auseinander.
}

Der G-BA ist für die Definition von Qualitätszielen und Qualitätsvorgaben in der gesetzlichen Krankenversicherung zuständig. Er setzt sich aus Vertretern der Krankenkassen, der Kassenärztlichen Bundesvereinigung, der Deutschen Krankenhausgesellschaft und Patientenvertretern zusammen. Krankenkassen, Ärzte und Krankenhäuser sind stimmberechtigt. Die Patientenvertreter haben nur beratende Funktion. Die meiste Arbeit wird in Arbeitsausschüssen geleistet, in denen sich die Krankenkassen von Mitgliedern des Medizinischen Dienstes vertreten lassen, um den notwendigen medizinischen Sachverstand einzubringen. Die Kassenärztliche Bundesvereinigung besteht überwiegend aus Ärzten, die in der Kassenärztlichen Bundesvereinigung angestellt sind, aber auch aus Mandatsträgern aus regionalen KVen. Die Deutsche Krankenhausgesellschaft bringt sich mit Mitgliedern der Geschäftsführung und einzelnen ausgesuchten Krankenhausärzten ein. Diese sogenannten Bänke versuchen damit den ihnen zur Verfügung stehenden medizinischen Sachverstand bei der Erarbeitung der Qualitätsvorgaben zu bündeln. Meist ist es bei der hochspezialisierten Medizin, über die entschieden werden soll, aber nötig, zusätzlich Gutachter von den Fachgesellschaften hinzuzuziehen. Berufsverbände werden sehr selten dabei berücksichtigt. Im Plenum des Gemeinsamen Bundesausschusses sitzen die Repräsentanten der verschiedenen Bänke. Sie befinden in der Regel über entscheidungsreife Vorlagen zusammen mit den unparteiischen Mitgliedern des Gemeinsamen Bundesausschusses, die bei strittigen Fällen über ihre Stimme die Entscheidung herbeiführen.

Das Gremium hat in der Vergangenheit zahlreiche Qualitätsziele definiert, aber meist methodenbezogen. Die Beschlüsse sollen eine qualitativ hochwertige Versorgung der Patienten sichern. Ziel ist es dabei, über eine Nutzenbewertung zu einer sachgerechten Entscheidung zu kommen.

Die Beschlüsse haben für die Leistungserbringer, Vertragsärzte wie Krankenhäuser, in der Regel auch wirtschaftliche Folgen. Die Qualitätsvorgaben beziehen sich meist auf Innovationen, deren Honorierung insbesondere für Krankenhäuser lukrativ erscheint. So können durch die Qualitätsvorgaben bestimmte Leistungserbringer ausgeschlossen werden, mit ggf. erheblichen wirtschaftlichen Folgen. Der Gemeinsame Bundesausschuss muss deshalb dafür sorgen, dass absolut rechtssichere Beschlüsse gefasst werden, die auch vor einem Gericht Bestand haben. Die Entscheidungen müssen schlüssig und nachvollziehbar sein.

Der Gemeinsame Bundesausschuss bedient sich bei der Beschlussfassung wissenschaftlich gesicherter Daten. Nach einer ausführlichen, in der Regel weltweit durchgeführten Literaturrecherche werden die zur Verfügung stehenden Publikationen nach den Kriterien der evidenzbasierten Medizin eingestuft. Bei einem stringenten Vorgehen führt dies zu einer restriktiven Entschei- 
dungspraxis, da viele Fragestellungen nicht nach evidenzbasierten Kriterien untersucht worden sind. Es kommt auch vor, dass die Frage nicht lupenrein in den wissenschaftlichen Veröffentlichungen gestellt und damit beantwortet wird. Um praktikable Qualitätsvorgaben zu erarbeiten, muss man somit auf Expertenmeinungen zurückgreifen, die in der Evidenz niedrig bewertet werden. Die Irrtumswahrscheinlichkeit ist hier größer als beim Vorliegen von hochwertigen Studien. Insbesondere bei Details in den Qualitätszielen fehlt deshalb häufig eine gesicherte wissenschaftliche Begründung. Die Bedeutung der evidenzbasierten Medizin für die Entscheidungspraxis ist zwar hoch, es bleibt aber immer noch viel Raum für eine subjektive Bewertung.

Der Gemeinsame Bundesausschuss versucht in dieser Gemengelage, die dadurch verursachte Beliebigkeit über fundierte Expertenentscheidungen einzugrenzen. Analysiert man manche Beschlüsse, so entdeckt man, dass offensichtlich nicht nur die Wissenschaft bei der Entscheidung Pate gestanden hat. Qualitätsziele werden sehr häufig auch

\section{Die Krankenkassen stehen von Anfang an Innovationen skeptisch gegenüber.}

zu Verteilungskämpfen innerhalb unseres Gesundheitswesens missbraucht. Die jeweilige Interessenlage spielt auch bei den gesetzlich vorgesehenen Mitgliedern des Gemeinsamen Bundesausschusses eine Rolle. Die Krankenkassen als Kostenträger stehen von Anfang an Innovationen skeptisch gegenüber. Sie befürchten, dass bei zugelassenen Innovationen zusätzliche Finanzmittel notwendig werden. Man versucht deshalb die Hürden für die Qualitätsziele besonders hoch anzusetzen. Dieses Vorgehen entlarvt, dass Qualitätsvorgaben auch zur Mengenbegrenzung von Leistungen benutzt werden. Die Vertragsärzte schließen sich der Meinung der Krankenkassen oft an und entscheiden ebenfalls restriktiv, meist auch aus wirtschaftlichen Gründen. Sie fürchten, dass eine neu eingeführte Leistung bei niedrigen Hürden in der Qualitätsvorgabe so häufig abgerechnet wird, dass eine Belastung der budgetierten Gesamtvergütung ein- tritt. Sie sind deshalb auch eher an einer Einschränkung der Mengenentwicklung interessiert.

In einer besonderen Position befinden sich die Krankenhäuser. Sehr oft werden durch das DRG-Abrechnungssystem Innovationen über das NUB-System eingeführt, ohne dass der Gemeinsame Bundesausschuss hierzu bereits Qualitätssicherungsmaßnahmen beschlossen hat. Die Krankenhäuser sind deshalb an einer eher allgemein formulierten Definition von Qualitätszielen interessiert. Sie wollen verhindern, dass die tägliche Anwendungspraxis durch neue Vorschriften eingegrenzt wird. Auch die hinzugezogenen Gutachter, die sehr oft aus Fachgesellschaften kommen, sind nicht frei von wirtschaftlichen Zwängen. Insbesondere überzogene Strukturvorgaben führen zu einer Ausgrenzung von Leistungserbringern im ambulanten und stationären Bereichen und sollen zu einer Zentrierung auf Groß-Krankenhäuser beitragen. Somit werden die ausgearbeiteten Qualitätsvorgaben auch zu einem Mittel der Krankenhauspolitik.

Sollen die Qualitätsziele der ASV im Interesse der Patienten wissenschaftlich medizinisch begründet und zusätzlich praktikabel sein, müssen sich alle Beteiligten nur an medizinischen Daten und Notwendigkeiten orientieren. Wirtschaftliche Zwänge dürfen bei der Bewertung keine Rolle spielen. Es besteht ansonsten die Gefahr, dass das Ziel der ambulanten spezialfachärztlichen Versorgung, eine hohe Qualität in Krankenhaus und Praxis zu erreichen, verfehlt wird. Die ambulante spezialfachärztliche Versorgung ist auf Kooperation und nicht auf Verteilungskampf ausgerichtet. So könnte es durchaus Sinn machen, dass die Beteiligten an einer Entscheidung in den Stellungnahmen wirtschaftliche Konflikte benennen müssen, so wie wir dies bei den Sponsorenangaben bei wissenschaftlichen Publikationen kennen.

Es soll hier nicht der Eindruck entstehen, dass sich der Gemeinsame Bundesausschuss wenig an wissenschaftlichen Kriterien orientiert - im Gegenteil. Die oft zögerliche Entscheidungspraxis wird aber durch wirtschaftlich orientierte Diskussionen unnötig in die Länge gezogen.

Die Definitionen der Qualitätsziele stellen bei dem derzeitigen Katalog des
\116 b hohe Anforderungen an den Gemeinsamen Bundesausschuss. Es handelt es sich um Vorgaben für komplexe Krankheitsbilder, in denen zahlreiche unterschiedliche Behandlungen und Untersuchungsverfahren eingearbeitet werden müssen. Es geht nicht wie seither um Erstellung von Qualitätszielen für einzelne Operationen oder Behandlungsverfahren, die Aufgabe ist durch die Erweiterung auf ganze Krankheitsbilder wesentlich komplexer geworden.

\section{1. Überprüfung der Überweisungsdiagnose zur ambulanten spezialfachärztlichen Versorgung}

Nach der Beschlussfassung des Gemeinsamen Bundesausschusses vom 21.03.2013 ist festgelegt, dass ein $\mathrm{Pa}$ tient in der ambulanten spezialfachärztlichen Versorgung nur behandelt werden kann, wenn eine Überweisung aufgrund einer gesicherten Diagnose erfolgt. Der zuweisende Arzt muss den ASV-Arzt umfassend über Vorgeschichte und Vorbefunde informieren, damit dieser überprüfen kann, ob mit den seitherigen Untersuchungsergebnissen die Diagnose auch tatsächlich sicher ist. Es ist zu erwarten, dass bei einem großen Teil der überwiesenen Patienten über die eingereichten Befunde hinaus zusätzliche Untersuchungsverfahren notwendig sind. Dies kann von modernen Schnittbildverfahren in der Bildgebung bis hin zu einer Sicherung der Diagnose durch eine Histologie reichen. Der Gemeinsame Bundesausschuss wird deshalb bei seinen allgemeinen Bestimmungen noch festlegen müssen, ob das ASV-Team diese Leistungen selbst erbringen kann oder ob sie in der Regelversorgung veranlasst werden müssen. Es ist auch denkbar, dass der Patient mit entsprechenden Vorgaben wieder zu seinem überweisenden Arzt zurücküberwiesen wird. Hier muss noch eine möglichst patientenfreundliche Lösung gefunden werden.

\section{Schweregrad der Erkrankung}

Der Gesetzgeber hat im Vergleich zu dem früheren $\mathbb{S} 116 \mathrm{~b}$ den Leistungskatalog weiter eingegrenzt, in dem er vorschreibt, dass es sich um einen schwerwiegenden Verlauf der genannten Erkrankung handeln muss. Leichte Verläufe müssen von schwerwiegenden 
abgegrenzt werden. Dazu hat der Gemeinsame Bundesausschuss für jede einzelne Erkrankung griffige Kriterien zu definieren. Dies ist aufwändig und schwierig. Neben dem jeweiligen Stadium der Erkrankung müssen zusätzliche Aspekte berücksichtigt werden, z. B. Begleiterkrankungen oder Eingriffe, die einen Verlauf erschweren können. Man wird deshalb nicht umhin kommen, neben einer Stadieneinteilung der zu beurteilenden Erkrankung auch weitere Details in der Bewertung zu berücksichtigen. Die Definition „schwerwiegend" ist aus medizinischer Sicht sehr unglücklich. Der Gesetzgeber wäre gut beraten, diesen eher schwammigen und schwer definierbaren Begriff wieder zu streichen.

\section{Behandlungsverlauf}

Mit Aufnahme in die ambulant spezialfachärztliche Versorgung muss ein Behandlungsziel definiert werden. Dies erscheint sinnvoll, um die Behandlungsergebnisse bewerten zu können. Während des Verlaufs wird es notwendig sein, unterschiedliche therapeutische und diagnostische Verfahren anzuwenden. Bei onkologischen Erkrankungen muss u. a. bei der Therapie zwischen Zytostatika, Strahlentherapie und operativer Behandlung differenziert werden. Die dazu notwendigen diagnostischen Verfahren reichen von einfachen sonographischen Untersuchungen bis hin zu aufwendigen tomographischen Kontrollen und weiter bis zur PET. Die Entscheidungen sind medizinisch zu begründen, wobei insbesondere bei der Diagnostik eine Angabe zur therapeutischen Konsequenz gefordert werden muss. Es bietet sich an, das Modell „Clinical Pathway“ als Vorlage zu benutzen. Dabei ist es ausdrücklich erlaubt, von den vorgesehenen Behandlungsabläufen abzuweichen, um individuell notwendige Entscheidungen zu treffen. Das Abweichen muss medizinisch aber ausreichend begründet werden und muß bei der Qualitätskontrolle nachvollzogen werden.

Andererseits dürfen die Vorgaben nicht so detailliert formuliert werden, dass Innovationen, die noch als Variante von gesicherten Behandlungsverfahren angesehen werden können, wieder zu einer neuen Entscheidungsvorlage für den Gemeinsamen Bundesausschuss führen. Die Formulierungen des Clinical Pathways müssen eindeutig aber ausreichend flexibel sein. In der Diskussion über die Qualitätssicherungsverfahren bei den einzelnen Krankheitsbildern wird sich herausstellen, ob die Vorgabe Clinical Pathway praktikabel sein wird.

\section{Verlaufsdokumentation}

Die von dem ASV-Team getroffenen Entscheidungen über die verschiedenen Behandlungsabläufe müssen klar dokumentiert sein. Mindestens einmal im Jahr ist eine zusammenfassende Dokumentation und Bewertung der therapeutischen und diagnostischen Maßnahmen sinnvoll, auch um das Therapieergebnis mit den ursprünglich definierten Behandlungszielen zu vergleichen.

\section{Weiterentwicklung der Qualitätsziele}

Der bürokratische Aufwand wird deshalb erhöht, weil insbesondere bei onkologischen Erkrankungen kurzfristig neue Therapieansätze eingeführt werden. Für einzelne Fachgebiete sollten Expertengruppen gebildet werden, die die einschlägige Literatur sammeln und

Die von der ASV geforderte krankheitsbezogene Definition ist die hohe Schule bei der Erarbeitung von Qualitätszielen.

bewerten und die Entscheidungsgremien informieren, wenn eine Änderung der Qualitätsvorgaben erforderlich wird. Dafür wird eine konsequente KostenNutzen-Bewertung einzuhalten sein. Die erarbeiteten Vorlagen werden nicht ohne Einfluss auf die Pharmakotherapie dieser Krankheiten bleiben.

\section{Register, Versorgungsforschung}

Die Definition der Qualitätsziele von Behandlungszielen und die Qualitätsvorgaben erlaubt eine Bewertung der Ergebnisqualität, die in einem Register erfasst werden sollte. Die ambulant spezialfachärztliche Versorgung wird einen
Überblick über die Behandlungsformen ihre Häufigkeit und ihr Ergebnis ermöglichen. Dies hat es in unserem Gesundheitswesen in dieser umfassenden Form seither noch nicht gegeben.

Die von der ambulant spezialfachärztlichen Versorgung geforderte krankheitsbezogene Definition ist somit die hohe Schule bei der Erarbeitung von Qualitätszielen. Man kann nochmals dem Gemeinsamen Bundesausschuss und seinen Beratern aus den wissenschaftlichen Gesellschaften empfehlen, nur nach medizinischen Gesichtspunkten zu urteilen und wirtschaftliche Aspekte bei den Qualitätszielen in der Bewertung außen vor zu lassen. Diese werden sicher an anderer Stelle, z. B. bei Honorarverhandlungen und in Bewertungsausschüssen ausreichend Berücksichtigung finden.

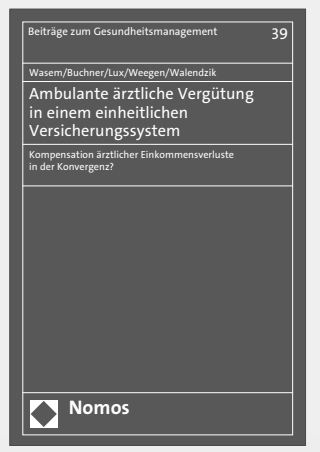

Ambulante ärztliche Vergütung in einem einheitlichen Versicherungssystem

Kompensation ärztlicher Einkommensverluste in der Konvergenz?

Von Jürgen Wasem, Florian Buchner, Gerald Lux, Anke Walendzik und Lennart Weegen 2013, 104 S., brosch., 16,- $€$ ISBN 978-3-8487-0489-7 (Beiträge zum Gesundheitsmanagement, $B d$. 39)

www.nomos-shop.de/21148

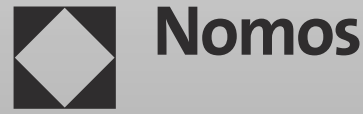

